SDCs and ICCs are presented in Table 1. ICCs for mean status scores are good to excellent, with the lowest ICCs occurring in the cervical segment. ICCs for change scores vary greatly, the lowest ICCs being in the lumbar segment, the highest in the cervical segment. The proportion of patients with change larger than 0 , larger than the positive SDC and larger than the negative SDC are given in Table 2 per reader and for consensus between readers. The largest percentages of change are seen in the thoracic segment.

Conclusion: Reliability of facet joint ankylosis progression was good in the whole spine but poor in the lumbar segment where little progression was seen. Over two years, a fair number of patients had progression of facet joint ankylosis with most progression occurring in the thoracic spine. These results show that using whole spine IdCT, progression of facet joint ankylosis can be studied in r-axSpA patients.

Disclosure of Interests: Rosalinde Stal: None declared, Floris A. van Gaalen: None declared, Alexandre Sepriano: None declared, Juergen Braun Shareholder of: AbbVie, BMS, Celgene, Chugai, Merck, Novartis, Pfizer, UCB, Grant/research support from: AbbVie, BMS, Celgene, Chugai, Merck, Novartis, Pfizer, UCB, Grant/research support from: Abbott, Bristol Myers Squibb, Celgene, Celltrion, Chugai, Johnson \& Johnson, MSD, Novartis, Pfizer, Roche, UCB Pharma, Grant/research support from: AbbVie, BMS, Celgene, Chugai, Merck, Novartis, Pfizer, UCB, Grant/research support from: Abbvie (Abbott), Amgen, Baxter, Biogen, BMS, Boehringer, Celgene, Celltrion, Centocor, Chugai, Hexal, Janssen, Lilly, Medac, MSD

Table 1: Facet joint ankylosis prevalence, interreader reliability and progression in patients with $r$-axSpA with at least one syndesmophyte

\begin{tabular}{|c|c|c|c|c|}
\hline \multicolumn{5}{|c|}{\begin{tabular}{|ll} 
& mean (SD) range \\
\end{tabular}} \\
\hline Whole spine e & & & & \\
\hline $\begin{array}{l}\text { Baseline } \\
\text { Ban }\end{array}$ & $6.9(9.5) 0-34$ & $9.2(10.3) 0-32$ & $0.93(0.87-0.96)$ & \\
\hline Follow-up & $7.8(9.7) 0.34$ & $10.3(10.9) 0 \cdot 34$ & $0.91(0.82-0.95)$ & \\
\hline $\begin{array}{l}\text { Change score } \\
\text { Chat }\end{array}$ & $0.8(2.4)-8.4-6$ & $0.9(2.1)-4.2-6.3$ & $0.75(0.53-0.87)$ & \\
\hline \multicolumn{5}{|c|}{$\begin{array}{l}\text { Cervical excluding C5-T1 max score } 6 \\
\end{array}$} \\
\hline Baseline & $0.8(1.7) 0.6$ & $1.1(1.9) 0-6$ & $0.84(0.73-0.90)$ & \\
\hline Follow-up & $0.7(1.6) 0-6$ & $1.2(1.9) 0-6$ & $0.72(0.52-0.84)$ & \\
\hline Change score & $0.0(0.4) \cdot 2 \cdot 1$ & $0.0(0.4)-2 \cdot 1.2$ & $0.91(0.83-0.95)$ & 0.2 \\
\hline \multicolumn{5}{|c|}{\begin{tabular}{|l} 
Thoracic excluding T1-T2 max score 22 \\
\end{tabular}} \\
\hline Baseline & $5.1(7.1) 0-22$ & $6.4(7.4) 0-22$ & $0.91(0.85-0.95)$ & \\
\hline Follow-up & $6.0(7.2) 0.22$ & $7.3(7.9) 0.22$ & $0.90(0.82-0.94)$ & \\
\hline Change score & $0.9(2.5)-8.8-7$ & $0.9(2.3)-4.4-11$ & $0.56(0.21-0.75)$ & 2.7 \\
\hline \multicolumn{5}{|c|}{\begin{tabular}{|l|} 
Lumbar max score 10 \\
\end{tabular}} \\
\hline Baseline & $1.0(2.4) 0-10$ & $1.6(2.9) 0-10$ & $0.81(0.67-0.89)$ & \\
\hline Follow-up & $\begin{array}{l}1.1(2.5) 0-10 \\
1.10\end{array}$ & $1.8(3) 0-10$ & $0.85(0.73-0.92)$ & \\
\hline Change score & $0.0(0.8)-2 \cdot 3$ & $0.2(0.9)-2 \cdot 5$ & $0.10(-0.58-0.48)$ & 1.1 \\
\hline
\end{tabular}

Baseline and follow-up: $\mathrm{N}=52$ (reader 1), $\mathrm{N}=53$ (reader 2); whole spine change score: $\mathrm{N}=42$ (reader 1), $\mathrm{N}=44$ (reader 2), $\mathrm{N}=38$ (ICC); cervical segment change score: $\mathrm{N}=44$ (reader 1), $\mathrm{N}=45$ (reader 2), $\mathrm{N}=40$ (ICC); thoracic segment change score: $\mathrm{N}=50$ (reader 1 ), $\mathrm{N}=52$ (reader 2), $\mathrm{N}=50$ (ICC); lumbar segment change score: $\mathrm{N}=51$ (reader 1), $\mathrm{N}=52$ (reader2), $\mathrm{N}=51$ (ICC)

Table 2: Number of radiographic axSpA patients with progression of facet joint ankylosis during 2-year follow

\begin{tabular}{|l|r|r|r|r|}
\hline & SDC & Reader 1 N(\%) & Reader 2 N(\%) & Consensus N(\%) \\
\hline Whole spine & 2 & & & \\
Change $>$ SDC positive & & $12(28.6 \%)$ & $11(25 \%)$ & $6(15.8 \%)$ \\
Change $>$ SDC negative & & $1(2.4 \%)$ & $3(6.8 \%)$ & $1(2.6 \%)$ \\
Change $>0$ & & $13(30.1 \%)$ & $15(34.1 \%)$ & $6(15.8 \%)$ \\
\hline Cervical segment & 0.2 & & & \\
Change $>S D C$ positive & & $2(4.5 \%)$ & $3(6.6 \%)$ & $1(2.5 \%)$ \\
Change $>$ SDC negative & & $1(2.3 \%)$ & $1(2.2 \%)$ & $1(2.5 \%)$ \\
Change $>0$ & & $2(4.5 \%)$ & $3(6.6 \%)$ & $1(2.5 \%)$ \\
\hline Thoracic segment & 2.7 & & & \\
Change $>S D C$ positive & & $9(18 \%)$ & $8(15.4 \%)$ & $3(6 \%)$ \\
Change $>S D C$ negative & & $1(2 \%)$ & $2(3.8 \%)$ & $1(2 \%)$ \\
Change $>0$ & & $15(30 \%)$ & $15(28.8 \%)$ & $5(10 \%)$ \\
\hline Lumbar segment & 1.1 & & & \\
Change $>S D C$ positive & & $3(5.9 \%)$ & $4(7.7 \%)$ & $2(3.9 \%)$ \\
Change $>S D C$ negative & & $3(5.9 \%)$ & $1(1.9 \%)$ & $1(2 \%)$ \\
Change $>0$ & & $3(5.9 \%)$ & $6(11.5 \%)$ & $2(3.9 \%)$ \\
\hline
\end{tabular}

Whole spine: $\mathrm{N}=42$ (reader1), $\mathrm{N}=44$ (reader 2), $\mathrm{N}=38$ (consensus); cervical segment: $\mathrm{N}=44$ (reader 1), $\mathrm{N}=45$ (reader 2), $\mathrm{N}=40$ (consensus); thoracic segment: $\mathrm{N}=50$ (reader 1 ), $\mathrm{N}=52$ (reader 2), $\mathrm{N}=50$ (consensus); lumbar segment: $\mathrm{N}=51$ (reader 1 ), $\mathrm{N}=52$ (reader 2$), \mathrm{N}=51$ (consensus)
(Schering-Plough), Mylan, Mundipharma, Novartis, Pfizer (Wyeth, Hospira), Roche, Sanofi-Aventis and UCB, Consultant for: Abbvie (Abbott), Amgen, Baxter, Biogen, BMS, Boehringer, Celgene, Celltrion, Centocor, Chugai, Hexal, Janssen, Lilly, Medac, MSD (Schering-Plough), Mylan, Mundipharma, Novartis, Pfizer (Wyeth, Hospira), Roche, Sanofi-Aventis and UCB, Consultant for: AbbVie, BMS, Celgene, Chugai, Merck, Novartis, Pfizer, UCB, Consultant for: Abbott, Bristol Myers Squibb, Celgene, Celltrion, Chugai, Johnson \& Johnson, MSD, Novartis, Pfizer, Roche, UCB Pharma, Speakers bureau: AbbVie, BMS, Celgene, Chugai, Merck, Novartis, Pfizer, UCB, Speakers bureau: Abbvie (Abbott), Amgen, Baxter, Biogen, BMS, Boehringer, Celgene, Celltrion, Centocor, Chugai, Hexal, Janssen, Lilly, Medac, MSD (Schering-Plough), Mylan, Mundipharma Novartis, Pfizer (Wyeth, Hospira), Roche, Sanofi-Aventis and UCB, Speakers bureau: AbbVie, BMS, Celgene, Chugai, Merck, Novartis, Pfizer, UCB Monique Reijnierse Grant/research support from: Funding from the Dutch Arthritis Foundation.

The funding source had no role in the design and conduct of the study. Désirée van der Heijde Consultant for: AbbVie, Amgen, Astellas, AstraZeneca, Bristol-Myers Squibb, Boehringer Ingelheim, Celgene, Daiichi, EliLilly, Galapagos, Gilead, GlaxoSmithKline, Janssen, Merck, Novartis, Pfizer, Regeneron, Roche, Sanofi, Takeda, Union Chimique Belge, Xenofon Baraliakos Grant/research support from: AbbVie, Boehringer Ingelheim, Bristol-Myers Squibb, Celgene, Centocor, Chugai, Janssen, MSD, Novartis Pfizer Inc, Roche and UCB, Grant/research support from: AbbVie, Pfizer, Merck Sharp \& Dohme, UCB Pharma, Novartis, Consultant for: AbbVie Bristol-Myers Squibb, Boehringer Ingelheim, Celgene, Chugai, Janssen Biologics, Novartis, Pfizer, UCB Pharma, Galapagos, Speakers bureau: AbbVie, Chugai, Janssen, Novartis, Pfizer, UCB Pharma

DOI: 10.1136/annrheumdis-2019-eular.4713

\section{SAT0530 SPSAMRIS: A TIME AND RESOURCE SAVING ALTERNATIVE FOR DETECTION AND MONITORING OF DISEASE-RELATED JOINT CHANGES?}

Philipp Sewerin, Daniel Abrar, Ralph Brinks, Stefan Vordenbäumen,

Alexander Lautwein, Matthias Schneider, Benedikt Ostendorf, Christoph Schleich. Universitätsklinikum Düsseldorf, Düsseldorf, Germany

Background: Standardized imaging scores in MRI are barley used in daily clinical practice whereby the high expenditure (in terms of time and staff) is often given as a reason.

Objectives: To develop and evaluate a simplified version of the OMERACT Psoriatic Arthritis Magnetic Resonance Imaging Score (PsAMRIS).

Methods: 19 patients with PsA according to CASPAR classification criteria with inadequate methotrexate response received anti-TNF treatment and were assessed by $3 \mathrm{~T}$ MRI of the clinically dominant hand at baseline and after 6 months. Scoring was according to PsAMRIS. Items were reduced based on standard response mean (SRM) resulting in SPSAMRIS, which was compared to PSAMRIS by calculation of the total SRM and relative efficiency (RE) after bootstrapping.

Results: PsAMRIS subscore of MCP3, 4, and PIP4 resulted in the highest SRM (-0.07 each) and were therefore included in SPsAMRIS. SPsAMRIS had higher SRM compared to PsAMRIS $(-0.13$ vs. -0.02$)$ and a higher RE (29.46). PsAMRIS and SPsAMRIS highly correlated at baseline $(r=0.75 ; p<0.01)$ and follow-up $(r=0.64 ; p=0.01)$. Evaluation time was reduced from 471 to 142 seconds at baseline and from 478 to $133 \mathrm{sec}$ onds at follow-up $(p<0.001)$.

Conclusion: The abbreviated MRI scoring system for PsA-related changes in hands, SPSAMRIS, showed a strong correlation with the well-evaluated PsAMRIS. Hence, SPSAMRIS can be considered as a time and resource saving alternative for semi-quantitative evaluation and monitoring of PsA related joint-pathology.

Acknowledgement: The project was funded by a grant from the German "Bundesministerium für Bildung und Forschung"(BMBF), ArthroMark (01EC1009).

Disclosure of Interests: Philipp Sewerin: None declared, Daniel Abrar: None declared, Ralph Brinks: None declared, Stefan Vordenbäumen: None declared, Alexander Lautwein: None declared, Matthias Schneider Grant/research support from: GlaxoSmithKline and UCB Pharma for performing the LuLa-study., Speakers bureau: Chugai, Benedikt Ostendorf: None declared, Christoph Schleich: None declared DOI: 10.1136/annrheumdis-2019-eular.4841 